\title{
Modelling and Design Concepts for Electronic Oscillators and its Synchronization
}

\author{
Wolfgang Mathis ${ }^{*}$ and Jan Bremer
}

\begin{abstract}
Department of Electrical Engineering and Computer Sciences, Leibniz University of Hannover, D-30167 Hannover, Germany

Abstract: In this paper we consider some concepts of deterministic and stochastic dynamical systems and discuss its application to electronic oscillator design. It is shown that a systematic design concept can be developed that includes nonlinearities and noise in an intrinsic manner. Although this concept is only in a prototype stage essential steps are presented and can be illustrated partly by means of modern CMOS oscillator circuits for GHz applications.
\end{abstract}

Keywords: Oscillators, synchronization, entrainment, Andronov-Hopf bifurcation, stochastic differential equations, stochastic bifurcation.

\section{INTRODUCTION}

Although electronic oscillators belong to the very first electric circuits at the beginning of the twentieth century a complete systematic design concept for this class of electronic circuits is not available until now. One of the reasons is that the behavior of these dynamical circuits depends in an intrinsic manner on the nonlinearities of within the circuit and therefore we are confronted with nonlinear differential equations. The oscillatory circuit behavior is related from a mathematical point of view to the so-called limit cycles. However electronic oscillators are fascinating circuits in many sense because the progress in manufacturing technologies of electronic devices and circuits led to new challenges in oscillator modeling and new mathematical concepts for solving the descriptive equations of oscillators. In certain cases the behavior of an electronic oscillator should be influenced by the behavior of other electronic systems in a desired manner such that entrainment and synchronization effects arise. Therefore driven nonlinear oscillators and their descriptive equations have to be considered where even chaotic behavior can appear. From a physical point of view electronic oscillators can be interpreted as such systems where dissipative structures occur. Ilya Prigogine coined this phrase as a name for the patterns which self-organize in far-from-equilibrium dissipative systems and limit cycles are a special case of them; see Nicolis, Prigogine [1]. Such dissipative systems are nonlinear and have to be connected with a DC power supply for delivering energy into the system. Furthermore these systems interact with a heat bath where energy is dissipated (Fig. 1). Accordingly the fluctuations of the heat bath influence the oscillator as electronic noise. As a result electronic oscillators have to be modeled by driven nonlinear stochastic differential equations with limit cycle-

*Address correspondence to this author at the Department of Electrical Engineering and Computer Sciences, Leibniz University of Hannover, D30167 Hannover, Germany, E-mail: mathis@tet.uni-hannover.de type solutions. In most cases analytical solutions for this type of equations are not available and approximation concepts have to be developed. From this point of view electronic oscillator circuits are until now a source of inspiration for new mathematical and physical concept; see e.g. Guckenheimer [2].

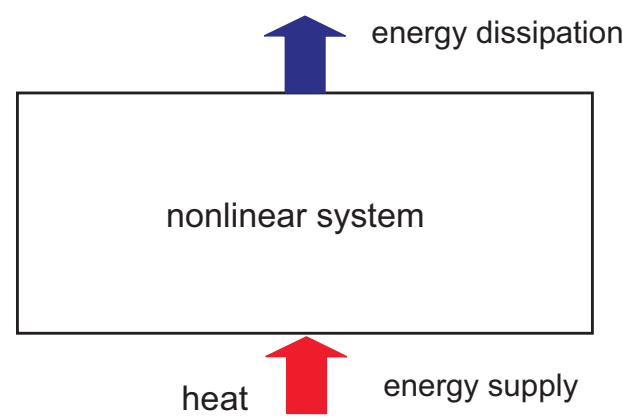

Fig. (1). Dissipative structure.

However for the oscillator circuit design not only approximative solutions of certain descriptive equations are needed since at the first stages of a design process only very few circuit parameters are known. Note that a circuit design concept consists of two steps where it is starting from the specifications of a circuit under design. These specifications are closely related to the solutions of the descriptive equations of the designed circuit. In a first design step the circuit architecture - circuit shape (O'Dell [3]) - has to be chosen whereas in the second step the free parameters of this circuit shape have to be determined. Therefore if not all circuit parameters are available it is even not possible to know whether the descriptive equations possess oscillatory or limit cycle solutions.

For this reason Mandelstam and Papalexi [4] developed in 1931 the concept of parametrized descriptive equations for oscillator circuits based on ideas of the French mathematician Henry Poincare. This concept was also the basis for the bifurcation theory of electronic oscillators; today it is called 
Andronov-Hopf theorem in the theory of dynamical systems. In contrast to the quantitative analysis of nonlinear differential equations this theorem studies these equations from a qualitative point of view. By means of the qualitative analysis we are able to consider the qualitative change of different types of solutions of nonlinear differential equations in dependence on certain circuit parameters. In the case of the Andronov-Hopf theorem the change from an equilibrium point to a limit cycle is explained. Although this theorem is known in electronic oscillator analysis since 1935 [5] (see Maggio et al. [6] for a more recent publication) it was never used for a systematic design process of electronic oscillators until recently; see Mathis \& Russer [7], Prochaska et al. [8]. As mentioned above the circuit description with parametrized equations is well suited for the second step of the design process and therefore the Andronov-Hopf theorem should be used in design processes for electronic oscillator circuits; a first concept idea was presented by Mathis [9].

In the following sections of this paper we will discuss a systematic design approach of electronic oscillators developed in my research group where all steps of this approach are based on advanced mathematical tools from dynamical systems theory. Before we discuss the details of the design approach a sketch of the history of electronic oscillator theory is presented. Then we discuss deterministic and stochastic aspects of oscillator analysis that are useful for the oscillator circuit design.

\section{A SKETCH ON THE HISTORY OF OSCILLATORS}

\section{A. Early Oscillator Circuits}

Since in 1895 Marconi showed for the first time that the laboratory arrangement of Hertz can be used for the wireless transmission of information along larger distances more powerful electrical arrangements were desired. Around 1900 several researchers (e.g. Thompson, Tesla, Slaby, Braun, von Arco and others) suggested improved versions of Marconi's arrangement. Probably it was a milestone as Duddel published his paper "On rapid variations in the current through the direct-current arc" [10] where he used results of the German physicist Simon [11]. A few years later Poulsen improved Duddel's oscillatory generator substantially from a technical point of view and as a result he presented in 1906 a new powerful arrangement with an arc as electronic device for wireless transmission of telephony signals. For further studies we refer to Blake [12] and Nesper [13].

Although the physical processes in arcs are rather difficult to understand at this time because of their electronic nature reasonable nonlinear models were developed by Kaufman, Duddel, Simon, Wagner and others. In the dissertation thesis of Wagner many aspects of such circuits were discussed [14]. Using these results Zenneck [15], a former co-worker of the above mentioned Braun, published in 1914 an interesting paper where he studied the start-up behavior of such RLC circuit including a nonlinear arc device. In contrast to his predecessors he described the behavior of the circuit by means of a nonlinear differential equation that described the energy (or power) balance. After solving this equation he got the approximative solution that is similar to the approximative of the van der Pol equation which was discovered several years later by van der Pol in the analysis of triode oscillators. From a mathematical point of view Zenneck's balance equation corresponds to an approximative first integral of the van der Pol equation. Zenneck's paper was also the first that studied the start-up behavior of oscillatory circuits in more details. Further details are considered in an other publication [16].

Circuits including sparks or arcs were the first successful generators for oscillatory currents. These electronic circuits had several disadvantages. Although engineers and physicists in the leading industrial companies (e.g. Marconi Comp., Telefunken, AT\&T, Western Electric Comp.) tried to improve these circuits by using interesting ingenious ideas the robustness of these arrangements as well as their transmitting power were rather restricted. Therefore a new generation of generators applied and now nonelectronic principle to get high-power oscillatory currents. For this purpose the static frequency doubling effect which was studied by Epstein, Joly and Vallauri was used for the development of rotating alternators (see Kühn [17], Meißner [18]). These generators had much better properties than the spark or arc circuits. Only the frequency of oscillatory waves generated by these electrical machines was restricted. However at this time transmitting stations worked with long waves. Around 1925 the situation was changed as the Heaviside layer was observed by nonprofessional users using transmitting stations with short waves. Within this range of frequencies rotating alternators cannot be applied. Moreover short waves did not need high-power transmitting stations such that the power can be reduced for these frequencies. As a conclusion electronic vacuum tubes were used to build generators with oscillatory behavior for powerful transmitting stations.

\section{B. Tubes and Oscillators}

First ideas for a new electronic device were published by Fleming in 1904. For the invention of his thermionic diode (or Fleming detector) he used research results in the area of emission and transport of electrons (Edison effect, Richardson, Wehnelt; see Johannsen [19]) in vacuum although the physical details were not well-known at this time. A modulation of the current in Fleming's thermionic diode was achieved by adding a grid. This was done by de Forest in 1906. This electronic device was called by de Forest as Audion [20]. In the same year v. Lieben presented a patent of triode type of amplifier valve [21] that was improved by him in a patent from 1910 together with Reisz and Strauß [22]. The audion as well as the "Lieben-valve were three-pole devices with cathode, grid and anode. In contrast to de Forest's audion the "Lieben-valve was filled with mercury. Therefore Lieben's valve was called "gas relay" and de Forest's "electron relay" (audion). This difference remained unclear in the following years and led to many discussions; see e.g. Armstrong [23] (p. 220) and Meißner [24] (p. 65). Further references can be found in a paper of Tucker [25]; see also Johannsen [19]. After the discovery of these triode valves it lasted further six years 
until first practical circuits were available. The first two classes of circuits were amplifiers and oscillators. Several groups in Germany, USA, United Kingdom, and Austria were participated in these activities and there were many relationships between these groups. Therefore it is difficult to solve the problem of priority with respect to the different electronic circuits; see e.g. Tucker [25], Johannsen [19], Barkhausen [26] (part II, pp.112), Meißner [24], and Skowronnek [27]. In 1913 Meißner presented a first high frequency transmitter including an electronic oscillator with a von Lieben tube (Fig. 2). It should be remarked that the principles of thermionic conduction were studied based on physical foundations by Langumir,

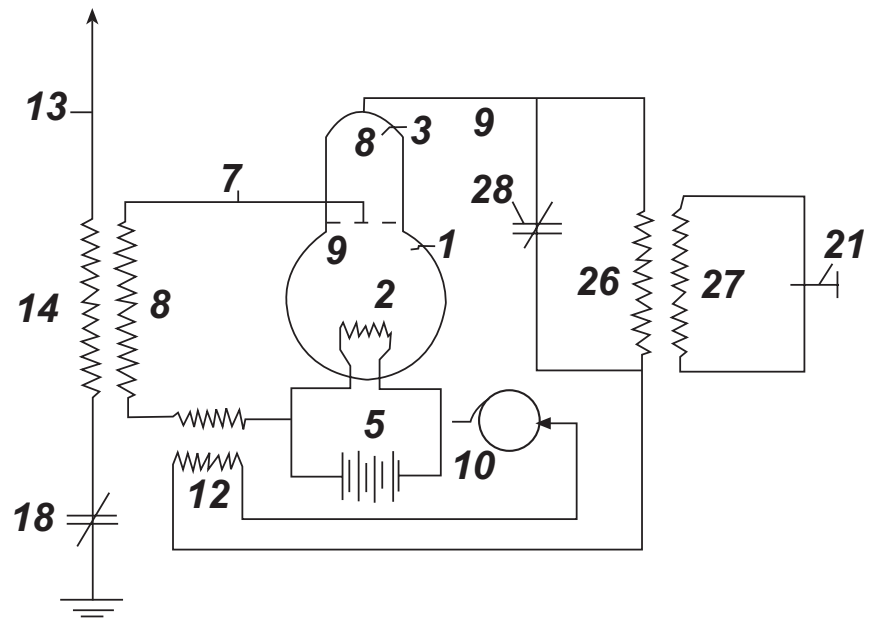

Fig. (2). Meißner's oscillator with a v. Lieben tube.

Dushman and Schottky between 1913 and 1915 (see Paschke [28] and Barkhausen [26] (p. 27)). In this paper we are interested in the descriptions of valves that use currents and voltages. This is suitable if we consider circuits from a network theoretical point of view. Therefore these electronic devices are described by characteristic curves (Armstrong [23]) or the corresponding German expression "Kennlinien" (Möller [29] (p. 326)).

A first example of an electronic oscillators with a valve which is the main subject of our paper was invented between 1912 and 1913 where the difference between De Forest's audion and the Lieben-Reisz valve is not considered (but see remarks of Meißner [24] (p. 65) and Hazeltine [30] (p. 98)). In agreement with Hazeltine [30] it seems that Meißner and Armstrong had similar ideas at the same time with respect to an oscillating circuit including a valve. The corresponding comments of Tucker [25] are a little bit obscure. Since the problem of the design of electronic circuits and especially oscillators with valves in order to transmit electromagnetic waves and receive them was a main subject in all military laboratories in all industrial states which are involved in the first world war many information became a secret. At the end of this war several electrical engineers and physicists published their results with a delay of one or more years (see corresponding remarks in the papers of Hazeltine [30], Barkhausen [26], Meißner [24] and others did not have an opportunity to publish it, just like Colpitts or Hartley). Hazeltine [30] (p. 98) gave some more references to interesting collections of oscillating circuits. Obviously at the end of the first world war many different oscillator circuits were known and several authors began to publish their theoretical results about this interesting class of electronic circuits.

\section{Descriptive Equations for Electronic Oscillators}

Probably the first theoretical paper about electrical oscillators with a valve was published by Vallauri [31] which was published in German some months later [32]. In this paper Vallauri used a linearization of the characteristic curves for the anode current and the grid voltage. As a result he received a linear theory of oscillators where he assumed that a sinusoidal oscillatory behavior already exists. By means of this approach Vallauri got "the exact determination of the conditions for oscillations in an audion circuit". After the publication of this paper many othe authors presented results that are more or less equivalent. These results were different in modifications of the valve model or the decomposition and its interpretation of the linearized oscillator circuit. We would like to mention only the comprehensive papers of Hazeltine [30], Heising [33] and Barkhausen [26]. The different approaches were compared by Albersheim [34].

Unfortunately it was known already before 1920 that a linear theory cannot be complete for describing all aspects of electronic oscillators. Whereas the conditions of oscillatory behavior can be derived by a linear model a nonlinear model is essential to determine the amplitude of these oscillators. This statement was given by Möller [29] in a very similar manner (p. 331). Based on the idea of the feedback principle for the functionality of electronic oscillators this author developed a theory for these circuits that used again the idea of a power balance equation (just like Zenneck in 1914 for the case of oscillator circuits with an arc). For this purpose Möller developed a concept that takes into consideration only the first harmonics of the oscillatory behavior and a nonlinear differential equation for the circuit was not derived. As a result he got the method of the "oscillatory characteristic" (in German "Schwingkennlinie") that can be interpreted now as a variant of the harmonic balance or averaging method. Some remarks to the history of this methods can be found in the monograph of Sanders and Verhulst [35] (pp. 181). Again other authors presented similar approaches that correspond to the fundamental mathematical problem of nonlinear oscillatory systems or nonlinear differential equations.

In contrast to Möller's approach van der Pol [36] derived in his doctorate thesis for the first time a nonlinear differential equation for an oscillatory electrical circuit and especially for an electronic oscillator circuit including a triode valve. Furthermore he was able to apply a special perturbation method that resulted in a solvable nonlinear differential equation. It was a variant of an averaging method (Lagrange's secular perturbation method) that was known to van der Pol from his studies in physics. In his famous paper from 1920 he states that the equation under consideration "is closely related to some problems which arise in the analytical treatment of the perturbation of planets by other 
planets." The differential equation of van der Pol became an eminent impact to a new mathematical discipline "nonlinear oscillations" and at the end the mathematical theory of dynamical systems. On the other hand we have to emphasize that although interesting from a theoretical point of view van der Pol's equation was not useful in practical situations at this time for two reasons: 1) Graphical differentiations of higher order arise. 2) Only in the simplest cases the perturbation method leads to a solvable differential equation. In other cases only the steady amplitudes can be calculated. Although Möller's method leads also to tedious calculations an semi-analytical approach was presented by Joos [37]. This author started with a good analytical approximation of the characteristic curves in form of an arctan-function and as a result he got another kind of oscillatory characteristics that did not include circuit parameters or valve constants. But also this method did not become popular.

Between 1920 and 1929 only very few scientific groups tried to develop new impacts to the theory of electrical oscillators. One of the few exceptions were van der Pol and Appleton. They published very interesting papers about the entrainment problem, forced oscillations and on relaxation problems as well as other aspects of nonlinear oscillators. The main results of these authors are contained in van der Pol's review paper from 1934 [38].

A new era of oscillator theory began as the Russian school of Mandelstam and Papalexi entered this area. Although Papalexi had published a book about "The Theory of Oscillators with Electronic Valves" in 1922 and Mandelstam was already a well-known scientist who was involved also in aspects of wireless transmission of electromagnetic waves they considered electronic oscillators from a new point of view. In 1929 Andronov who had Mandelstam as his academic teacher published a brief paper [39] where he applied Poincare's theory of limit cycles to the van der Pol equation. Another paper together with Witt [40] followed in 1930. In a further paper [41] these two authors showed that Poincare's theory is useful for studying rather difficult aspects of the entrainment effect in nonlinear oscillations. By means of these results it could be shown that the theory of limit cycles of Poincare was a suitable framework to study problems in nonlinear oscillations. Another important step towards an unified theory of nonlinear oscillations was the introduction of parametrized nonlinear differential equations and again Poincare's ideas were used. Mandelstam and Papalexi showed [4] that first aspects of a theorem that is now called Andronov-Hopf theorem and describes a bifurcation of a limit cycle from a static equilibrium point. These ideas became a big impact in Russia for the theory of nonlinear oscillations and several very active groups studied many aspects of this problem. The references of the famous book of Andronov, Witt and Chaikin [42] first published in 1937 illustrates this exciting area. In a review paper from 1935 Mandelstam, Papalexi, Andronov, Chaikin, and Witt [5] presented a complete concept of nonlinear oscillations that contains already almost all modern aspects of this theory; see also Mathis [43] and in particular the papers of Bissell [44], Aubin, Dalmedico [45] and Pechenkin [46]. It should be mentioned that at the same time Krylov and Bogoliubov [47] developed the mathematical foundations of a perturbation theory for oscillatory differential equations that is known now as averaging method or harmonic balance. It lasted more than 40 years until this subject was considered by Mees and Chua [48] in 1979. Afterwards Andronov-Hopf bifurcation became an essential subject in the theory of electrical circuits (e.g. Mathis [16]). It should be remarked that in higher dimensional systems $(d>2)$ additional methods are needed; see section III-A.

\section{Entrainment and Synchronization}

In many situations an electronic oscillator does not work in an autonomous manner but in a driven mode where some interesting phenomena arise. Historically entrainment effects were observed for the first time by Huyghens; see e.g. Rosenblum et al. [49] and Pantaleone [50]. At the beginning of the twentieth century entrainment effects were observed in superhet receivers where the local oscillator was entrained by the frequency of the antenna signal. This behavior was first studied by Möller in 1919 [51, 52] and later on he called it "Mitnahme" (entrainment); see also Fack [53] for further references and historical remarks. Möller presented a first theoretical explanation based of his invention of the socalled "Schwingkennlinie" and he showed that entrainment is a nonlinear phenomenon; see e.g. Kurz, Mathis [54] for a simplified explanation of entrainment. In the following years several other authors studied the entrainment phenomenon where more involved concepts from the theory of nonlinear differential equations were applied; we mention the papers of Appleton, van der Pol [55] and Andronov, Witt [41] (see also Mathis [56]). Although the principle behavior of the entrainment phenomenon was solved around 1930 Rjasin [57] published an interesting paper with further details where the transient behaviors were considered. At this time the entrainment behavior was interpreted as a disturbance without any applications. It should be remarked that acoustic entrainment effects were already observed by Rayleigh [58] in the nineteenth century; see also Chaikin [59]. Although the entrainment phenomenon was considered as a disturbance some engineers developed applications using it. One of the first was de Bellescise [60] in 1932 but other suggestions in measurement instrumentations came from Kaden and Reynauld (see Kirschstein [61]). One of the first major applications was the synchronization of vertical and horizontal scan in TV receivers. In 1938 Urtel [62] found out that entrainment can be interpreted as a control process where the frequency of the entrained oscillation is controlled in such a manner that the phase between this oscillation and the controlling oscillation is constant. Therefore Urtel observed similarities to the PLL concept for the first time, but this concept was unknown to him.

\section{DETERMINISTIC ASPECTS OF OSCILLATOR DESIGN}

\section{A. The Andronov-Hopf Bifurcation}

Although the descriptive equations of oscillatory circuits are in general of the type of the so-called differential algebraic equations (DAEs [43]) we will consider only those 
oscillator models which can be described by explicit ordinary differential or state-space equations

$\dot{x}=F(x), \quad F: \Re^{n} \rightarrow \Re^{n}$

As already mentioned above we do not consider transient analysis problems but (stable) asymptotic solutions of (1) and especially limit cycles in oscillator circuits. This kind of solutions can exist only if the circuit is described by nonlinear differential equations. Note that in textbooks of circuit theory and design Barkhausen's criteria or alternative methods are used in order to determine the frequency and something like the "amplitude" of sinusoidal oscillators. However these methods are based on the linearized descriptive equations of oscillator circuits and therefore the question about the sense of these methods arises. The answer to this question was given by the author of this article in the year 2000 [9] by

means of the Hartman-Grobman theorem. This theorem says that the dynamics of a nonlinear system in the neighborhood of an equilibrium point is equivalent to the dynamics of its associated linearized system only if it is a so-called hyperbolic system where the Jacobian matrix has only eigenvalues with nonzero real part; see also Mathis [63]. In this case the dynamical behavior is exponential. Only if we have a non-hyperbolic system where eigenvalues with zero real parts occur we can expect a more complex dynamical behavior - e.g. limit cycles. Since the Barkhausen-like criteria leads to a pair of eigenvalues on the imaginary axes a necessary condition for a stable limit cycles is fulfilled. This is the true reason behind the analysis of the associated linearized system in the sense of Barkhausen and others although the behavior of electronic oscillators is dominated by the nonlinearities.

In higher dimensions of the state space of a circuit no systematic methods for calculating limit cycles are available Papalexi, Mandelstam and Andronov developed the bifurcation approach for electrical oscillators using ideas from Poincaré's results. The main idea of these researchers was the embedding of (1) into a $\mu$-parametrized family of differential equations

$\dot{x}=F(x, \mu), \quad F: \Re^{n} \times \Re \rightarrow \Re^{n}$

and searching for a qualitative changing of asymptotic solutions within this family.

The Andronov-Hopf bifurcation theorem results in an efficient method for the analysis of limit cycles of autonomous one-parameter nonlinear ordinary differential equations of first order (2). By means of this theorem frequency and amplitude can be calculated in an approximative manner. Furthermore the stability of a limit cycle can be determined.

\section{Andronov-Hopf Theorem}

If the following conditions

Cl. the Jacobian matrix $\left.J_{F}(x, \mu)\right|_{\mu=\mu c} ^{x=x c}$ has a pair of conjugated complex eigenvalues $\lambda_{1,2}=\alpha(\mu) \pm j \omega(\mu)$ with the additional condition:
- $\left.\omega(\mu)\right|_{\mu=\mu c}=\omega_{C}>0$,

- $\left.\alpha(\mu)\right|_{\mu=\mu_{C}}=0$,

- $\quad d \alpha(\mu) /\left.d \mu\right|_{\mu=\mu c} \neq 0$

C2. all other eigenvalues have a negative real part $\mathrm{C} 3$. and the first Lyapunov coefficient $l_{l}$ is negative, that is, the equilibrium point is asymptotic stable for $\mu=\mu_{C}$, are fulfilled, then for bifurcation parameters $\mu>\mu_{C}$ a stable limit cycle exists and the oscillatory amplitude as well as the fundamental frequency can be approximated. (Fig. 3).

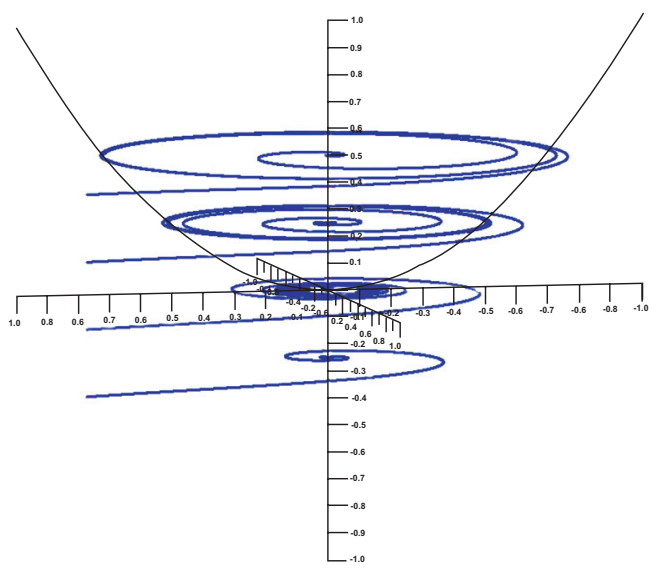

Fig. (3). Andronov-Hopf bifurcation.

\section{Bifurcation Analysis of a LC-Tank VCO}

In modern RF circuits for mobile communication systems so-called VCOs (Voltage Controlled Oscillators) for a few $\mathrm{GHz}$ belong to the most essential subcircuits. Therefore a design concept is needed where the necessary nonlinearity of VCOs has to be included. A first step to develop such a design concept based on the Andronov-Hopf theorem was the bifurcation analysis of LC-tank oscillators; see Mathis [9]. In order to design VCOs the voltage controlled capacitors have to be included in the analysis. Recently we present interesting semi-analytical results of a bifurcation analysis for a VCO in a $2.4 \mathrm{GHz}$ CMOS technology where the charge-based EKV-MOS model is used (Fig. 4) and Bremer, Zorn, Mathis [64]. It turns out that the reduced descriptive equations have the form

$\left(\begin{array}{c}\frac{d i_{L}}{d t} \\ \frac{d v_{t}}{d t}\end{array}\right)=\left(\begin{array}{cc}0 & -\omega_{C} \\ \omega_{C} & \alpha(\mu)\end{array}\right)\left(\begin{array}{l}i_{L} \\ v_{t}\end{array}\right)+\varepsilon f\left(v_{t}, i_{L}\right)$,

where

$\alpha(\mu)=-\frac{1}{C_{0}\left(V_{\text {tune }}\right)}\left[\frac{I_{\text {bias }} \sqrt{\mu_{n} C_{o x} W_{n}}}{2 \sqrt{I_{\text {bias }} L_{n}}}-\frac{1}{R_{t}}\right]$,

and 
$\omega_{C}=\frac{1}{\sqrt{C_{0}\left(V_{\text {tune }}\right) L_{t}}} ;$

$W_{n}$ is the width of the cross coupled pair and $L_{n}$ is its channel length. $C_{0}\left(V_{\text {tune }}\right)$ denotes the effective large signal capacitance of the VCO. It consists of the voltage dependent varactor capacitance $C_{v 0}\left(V_{\text {tune }}\right)$ and the parasitic capacitances of the other components of the VCO

$C_{0}\left(V_{\text {tune }}\right)=C_{v 0}\left(V_{\text {tune }}\right)+C_{\text {par }}$.

Obviously we can show that the condition $\mathrm{Cl}$ of the Andronov-Hopf bifurcation theorem is fulfilled if we have

$W_{n, C}=\frac{4 L_{n}}{\mu_{n} C_{o x} I_{\text {bias }} R_{t}^{2}}$

and also the transversality condition C1-3 and the stability condition are fulfilled. The limit cycle amplitude can be approximated by

$r=\sqrt{\frac{8}{3}} \frac{\sqrt{R_{t} I_{\text {bias }}\left(R_{t} I_{\text {bias }}-v_{n}\right)} \cdot v_{n}}{R_{t} I_{\text {bias }}}$,

where $v_{n}=\sqrt{\left(I_{\text {bias }} L_{n}\right) /\left(\mu C_{o x} W_{n}\right)}$.

\section{Example: Design of a $2.4 \mathrm{GHz}$ LC-Tank VCO}

In this section we show the application of our proposed design concept to a practical oscillator design. The desired specifications are shown in Fig. (5) (a $0.25 \mu \mathrm{m}$ RF CMOS technology from IHP (SGB25V) is used). An inductor with a series inductance of $L_{i, s}=11.1 \mathrm{nH}$ is chosen for the requested frequency range. Using our proposed model for the voltage dependent varactor capacitance [64] we are able to approximate the varactor dimensions. Fig. (6) shows an approximation of the frequency characteristic of the VCO in dependency of the varactor width $W_{v}$. Our calculations show, that as a first estimation a varactor width of $W_{n}=150 \mu \mathrm{m}$ is an appropriate choice for the requested frequency range (Fig. 5).
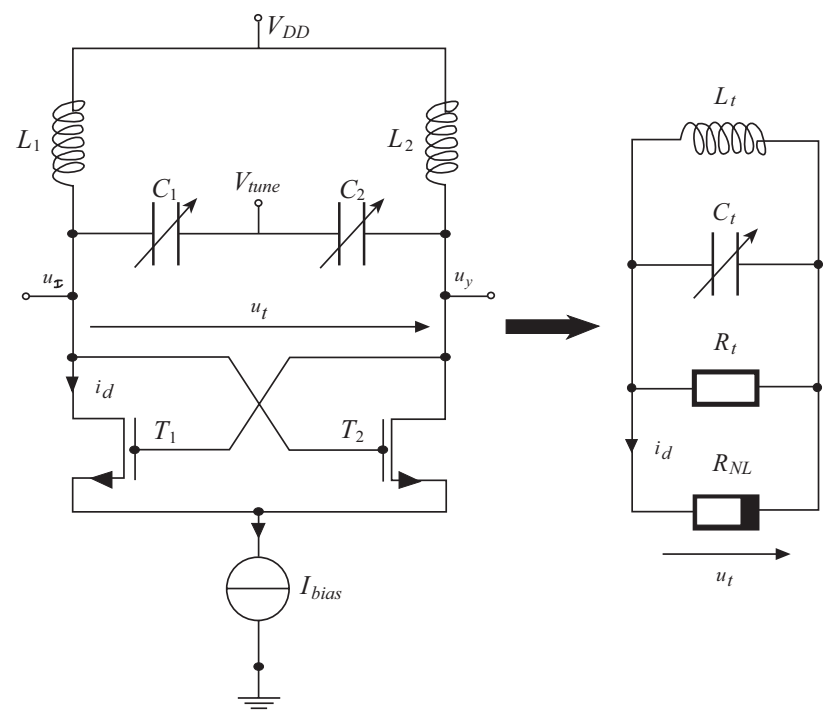

Fig. (4). LC-tank voltage controlled oscillator.
Setting $L_{v}$ to two times the minimum channel length is a good compromise between series resistance and $C_{v, \max } / C_{v, \min }$ ratio [65]. In order to minimize the parasitic resistance the length of the transistor pair $L_{n}$ is set to the minimum channel length. We set the value of $I_{\text {bias }}$ to the maximum value allowed by the specifications in order to maximize the output amplitude and optimize the phase noise characteristics of our VCO [66]. All design variables have been determined except the width $W_{n}$ of the transistor pair. It is guaranteed that a variation of the width of the transistor pair $W_{n}$ leads to a stable limit cycle as was shown in the previous section. Using expression (7) it is possible to calculate the needed width $W_{n}$. We find the bifurcation point and therefore the starting point of a stable oscillation at a parameter value of $W_{n}=2.03 \mu \mathrm{m}$. Increasing $W_{n}$ leads to an expansion of the limit cycle and accordingly to a rising of the oscillation amplitude (8) (Fig. 6).

The obtained design parameters are subsumed in Fig. (5). In order to validate the theoretical results we have simulated the VCO on circuit level in Cadence using a BSIM 3.4 MOS model (Fig. 7). The simulation results show that a stable oscillation is built up at a width of the transistor pair of $W_{n}=$ $3.05 \mu \mathrm{m}$, which is pretty close to our calculated bifurcation parameter. Calculation of the tank amplitude is only valid close to the bifurcation point and here the amplitude is too small to fulfill the required specifications. Increasing $W_{n}$ leads to a larger amplitude (see equation 8). We found that for a width of $W_{n}=4 \mu m$ the VCO has an amplitude of $v_{t}=$ $1.37 \mathrm{~V}$ which fulfills the requested amplitude specifications. Increasing the width $W_{n}$ beyond $4 \mu m$ does not increase the amplitude anymore. Until now we have only ensured that the VCO possesses a stable oscillation for a tuning voltage of $V_{\text {tune }}=0 \mathrm{~V}$. An increase of $V_{\text {tune }}$ leads to an increase of the tank capacitance because of the voltage-dependent varactor capacitance and thus to a higher VCO frequency. An increase of the frequency leads to a higher $R_{t}$ since the equivalent tank resistance $R_{t}$ is frequency-dependent. According to (7) the required width $W_{n}$ is inversely proportional to $R_{t}^{2}$, therefore it is ensured that the VCO designed at $\mathrm{V}_{\text {tune }}=0 \mathrm{~V}$ oscillates for the whole tuning range. The proposed design concept enables to find the minimum $W_{n}$ in order to built up a stable oscillation and additionally optimizes the amplitude of the VCO. Doing so the effect of the nonlinearity of the transistor pair and therefore the influence of the higher harmonics can be minimized. The design procedure leads to a VCO that possess a high spectral purity with a highly sinusoidal output signal.

\section{Higher Dimensional State Space Representation}

A higher dimensional state space representation allows the inclusion of other parasitic effects and structural enhancements in comparison to the 2-dimensional state space modeling. Examples of possible parasitic effects could be the nonlinear effects of the cross-coupled transistor pair or substrate effects for instance. An example for a structural enhancement that could be included in the modeling using higher dimensional state space equations is a filtering capacitance parallel to the current source (Fig. 8). In order to carry out the bifurcation analysis of an sinusoidal oscillator 


\begin{tabular}{|c|c|c|}
\hline Param. & Spec. & Achieved \\
\hline$f_{0}$ & $2.4 \mathrm{GHz}$ & $2.4 \mathrm{GHz}$ \\
\hline$V_{D D}$ & $2.5 \mathrm{~V}$ & $2.5 \mathrm{~V}$ \\
\hline$I_{\text {bias }}$ & $\leq 1 \mathrm{~mA}$ & $1 \mathrm{~mA}$ \\
\hline$T R$ & $\geq 20 \%$ & $34 \%$ \\
\hline$V_{t}$ & $\geq 1 \mathrm{~V}$ & $\geq 1.37 \mathrm{~V}$ \\
\hline$C_{\text {foad }}$ & $\geq 100 \mathrm{fF}$ & $100 \mathrm{fF}$ \\
\hline
\end{tabular}

\begin{tabular}{|c|c|c|}
\hline \multicolumn{3}{|c|}{ Transistor Pair } \\
\hline Param. & Maple & Cadence \\
\hline$W_{n}$ & $>2.03 \mu \mathrm{m}$ & $>3.05 \mu \mathrm{m}$ \\
\hline $\mathrm{L}_{n}$ & $250 \mathrm{~nm}$ & $250 \mathrm{~nm}$ \\
\hline $\mathrm{R}_{0}$ & $10 \mathrm{k} \Omega$ & $9.84 \mathrm{k} \Omega$ \\
\hline $\mathrm{C}_{\mathrm{N} M O \mathrm{~S}}$ & $3.97 \mathrm{fF}$ & $3.77 \mathrm{fF}$ \\
\hline
\end{tabular}

\begin{tabular}{|c|c|c|}
\hline \multicolumn{3}{|c|}{ Inductor } \\
\hline Param. & Maple & Cadence \\
\hline $\mathrm{L}_{\mathrm{i}, \mathrm{p}}$ & $11.16 \mathrm{nH}$ & - \\
\hline $\mathrm{C}_{\mathrm{i}, \mathrm{p}}$ & $16.6 \mathrm{fF}$ & - \\
\hline $\mathrm{R}_{\mathrm{i}, \mathrm{p}}$ & $1824 \Omega$ & - \\
\hline
\end{tabular}

\begin{tabular}{|c|c|c|}
\hline \multicolumn{3}{|c|}{ Varactor } \\
\hline Param. & Maple & Cadence \\
\hline$W_{v}$ & $150 \mu \mathrm{m}$ & $140 \mu \mathrm{m}$ \\
\hline $\mathrm{L}_{v}$ & $500 \mathrm{~nm}$ & $500 \mathrm{~nm}$ \\
\hline $\mathrm{R}_{v, \mathrm{p}}$ & $3222 \Omega$ & - \\
\hline $\mathrm{C}_{\mathrm{v} 0}$ & $378 \mathrm{fF}$ & - \\
\hline
\end{tabular}

Fig. (5). Specifications and design parameters for $V_{\text {tune }}=0$.
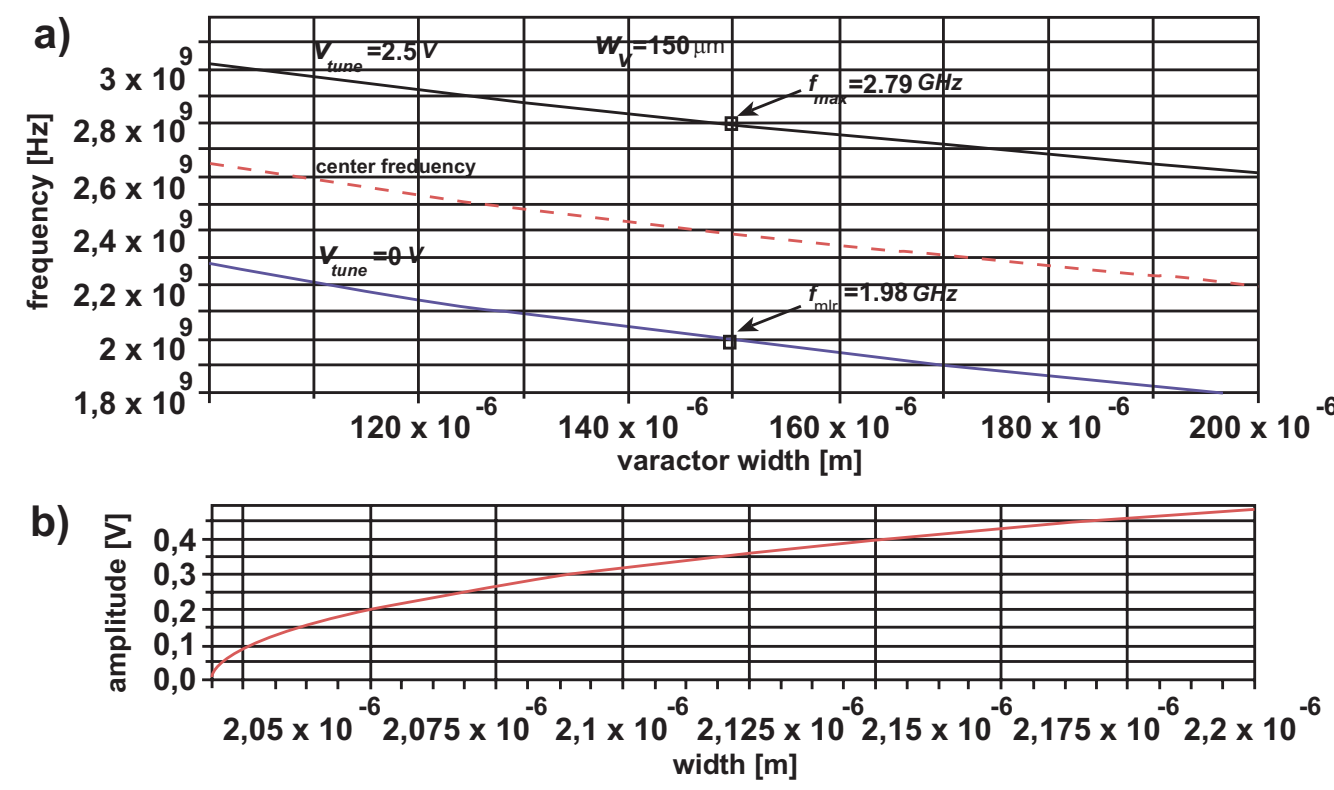

Fig. (6). Maple: (a) Estimated tuning range (b) Estimated amplitude.

with a higher dimensional state space in a more simple manner we have to calculate the corresponding center manifold and transform the descriptive equations to equations with a 2-dimensional state space. There are several important systematic techniques for the simplification of systems where simplification means that we can study the main aspects of a system on lower dimensional state space. The so-called center manifold reduction based on the center manifold theorem (e.g. Guckenheimer, Holmes [2]) takes advantage of the natural separation of timescales that occurs when some of the eigenvalues of a equilibrium point pass through zero. If a center manifold exists and no unstable solutions occur, the slow time dynamics dominate on this submanifold in the complete space and all other solutions rapidly approach this manifold (Fig. 9). To be more precise the vector field $F$ of $\dot{x}=F(x)$ is divided into a linear and a nonlinear part

$$
\begin{aligned}
& \dot{x}=A x+F_{c}(x, y), \\
& \dot{y}=B y+F_{s}(x, y),
\end{aligned}
$$

where the eigenvalues of $A$ have only zero real parts and of $B$ only negative real parts. The center manifold of this system is

$$
\begin{aligned}
& W^{C}(0):=\left\{(x, y) \in \Re^{C} \times \Re^{S} \mid y=h(x), h(0)=0,\right. \\
& D h(0)=0\},
\end{aligned}
$$

The function $h(x)$ is determined by

$B h(x)+F_{s}(x, h(x))=D h(x)\left(A x+F_{c}(x, h(x))\right)$

which is usually approximated in a power series. The simplified dynamics on the center manifold are described by the reduced system

$\dot{z}=A z+F_{c}(z, h(z)) \quad z \in \mathfrak{R}^{C}$.

Moreover the center manifold approach for simplifying dynamical systems has a nice geometric interpretation. Further investigations of the reduced system with analytical methods are well-known in system theory [2]. The following methods are predestinated for the use in common with center 

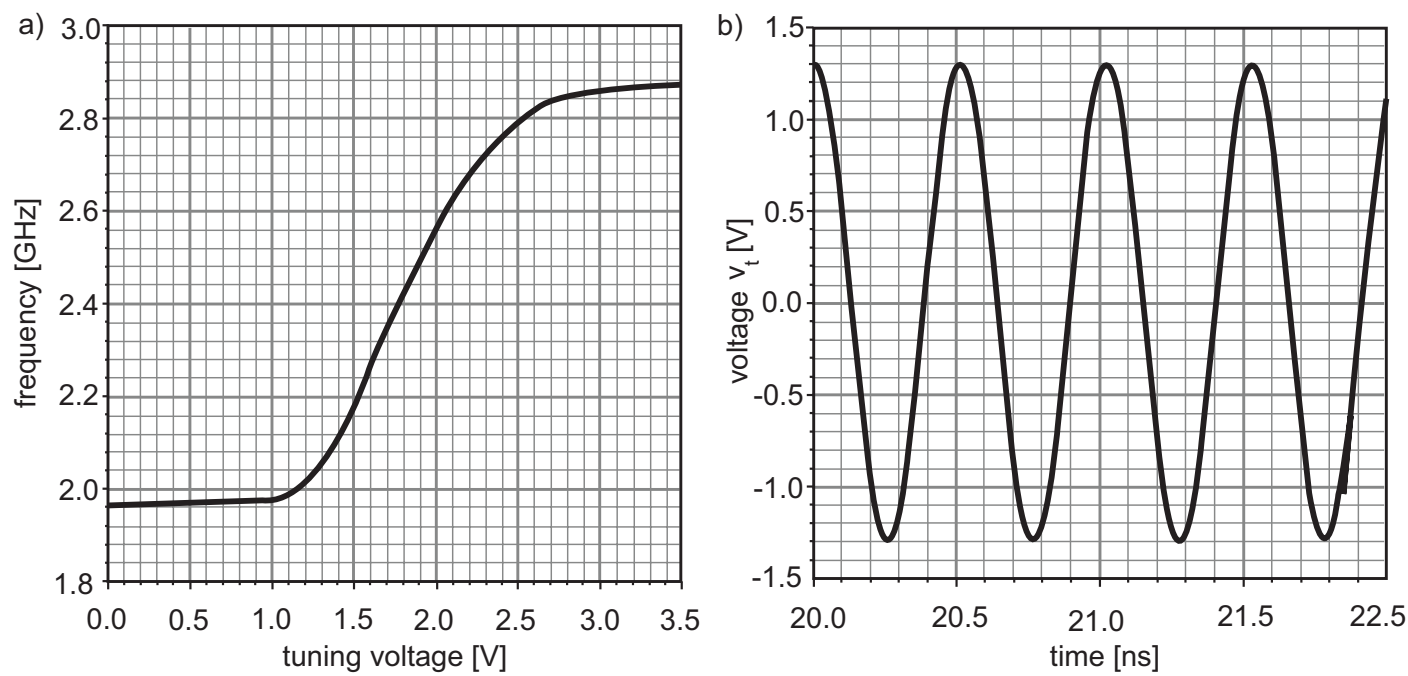

Fig. (7). Cadence: (a) Frequency characteristic (b) Transient output signal.

manifolds because they are formulated only in the 2dimensional case or easier to handle in that dimension:

-

Poincare-Normal-Form: A nonlinear system is transformed in a form showing the resonant (important) nonlinear terms [2]. The stability of equilibrium points can be estimated.

- Andronov-Hopf Bifurcation: A system's steady-state can be changed by parameter from a stable equilibrium point to a stable limit-cycle [2] where oscillation conditions can be identified.

- Amplitude-Angle-Form: A system with periodic (limit cycle) solutions is transformed into new coordinates representing amplitude and angle of the expected solution [67] where a separation of amplitude and angle is used.

- $\quad$ Averaged Form: Periodic solutions are averaged over a period of the basic oscillation [2]. Via Lie-series transformation nonlinear oscillating systems can be averaged with the help of computer-algebra [43]. This corresponds to a filtering-out of high-frequencyoscillations.

The use of computer-algebra for the presented methods is of high value, because they all depend on equivalence transformations [63]. These coordinate transformations are well done by symbolic manipulators. The approach can be illustrated by means of rather simple oscillator circuits (e.g. Colpitts and Hartley oscillators).

\section{B. Theoretical Aspects of Entrainment}

There are several theoretical approaches to analyze the entrainment phenomenon where in general we have to consider a nonlinear oscillator with excitation. Because the associated nonlinear differential equations cannot be solved and a perturbation concept is needed (see e.g. Mathis [43]). which characterizes the different approaches. Furthermore different kinds of differential equations with an oscillatory behavior can be presumed. The above mentioned papers of Appleton, van der Pol and Andronov, Witt as well as Rasjin are based on the van der Pol equation and applied van der Pol's perturbation method. Andronov and Witt studied the perturbation equations with the qualitative method of Poincaré but this method is rather complicated. A more simple method is Möller's "Schwingkennlinien" method (oscillatory characteristic; but see also Cassignol [68] and the generalized Barkhausen method) that based on a first order Fourier series approximation. A reformulation of this method based on a feedback model of an oscillator where an excitation is incorporated. As a result the amplitude of an oscillator can be determined.

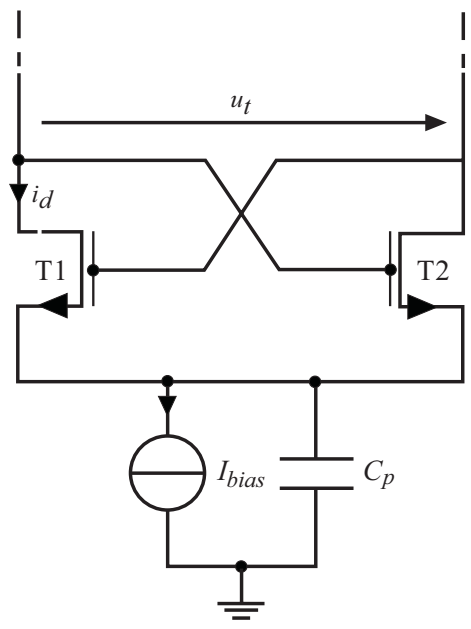

Fig. (8). LC-tank VCO with filter capacitance.

In more details an oscillator is modeled as a cascade of a nonlinear and a linear two-port where the output of the cascade is connected to its input. The nonlinear two-port is described by a nonlinear characteristic. The frequency characteristic of the linear two-port has a resonant form and only signals with the resonance frequency can pass. Therefore the corresponding term of the Fourier series of the output signal of the nonlinear two-port is necessary. In 
mathematical terms an input voltage of the nonlinear twoport is assumed

$U_{1}(t)=E e^{j(\omega t-\varphi)}$

and at its output voltage can be expressed by

$$
U_{2}(t)=F e^{j(\omega t-\varphi-\psi)} \text {. }
$$

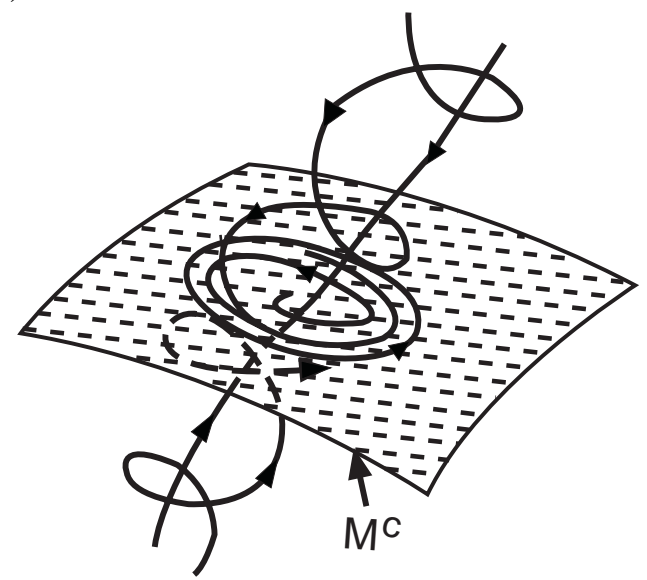

Fig. (9). Transient behavior near the center manifold.

From the feedback connection we have $U_{1}=U_{2}$ or $E=$ $F(E)$, where $F=F(E)$ is the nonlinear characteristic and the center frequency $\omega_{0}$ of the passband of the resonator filter has to be introduced. It can be shown that $\psi=\left(\omega-\omega_{0}\right) t_{0}$. If a graphical representation is available the amplitude of the oscillator can be determined in a graphical manner using $E=$ $F(E)$. In the case of entrainment an additional excitation voltage $U_{e}(t)=E_{e} e^{j \omega_{e} t}$ has to be included. Again a sinusoidal signal is assumed, but now $E$ and $\varphi$ are no longer constant but depend on time. In a similar way we derive the output voltage of the cascade of the two-ports

$U_{2}(t)=F(E) e^{-t_{o}(\dot{E} / F(E)) F^{\prime}(E)} e^{j\left(\omega_{e} t-\varphi-\left(\omega_{e}-\omega_{0}-\dot{\varphi}\right) t_{0}\right)}$,

where the momentum frequency is determined in a more complicated way and $\omega_{0}$ and $t_{0}$ are constants of the linear two-port. With feedback connection of the cascade $U_{2}=U_{I}$ $U_{e}$ we find

$$
F(E) e^{-t_{0}(\dot{E} / F(E)) F^{\prime}(E)}=\sqrt{E^{2}+E_{e}^{2}-2 E E_{e} \cos \varphi},
$$

where

$$
\tan \left(\left(\omega_{e}-\omega_{0}-\dot{\varphi}\right) t_{0}\right)=\frac{E_{e} \sin \varphi}{E-E_{e} \cos \varphi}
$$

In the case of a horizontal part of the "Schwingkennlinie" we have $F^{\prime}(E) \approx 0$ and therefore it results in

$$
F(E)=\sqrt{E^{2}+E_{e}^{2}-2 E E_{e} \cos \varphi} .
$$

If $E_{e}<<E$ eq. (19) can be simplified to

$\dot{\varphi}\left(t_{0}\right)=\left(\omega_{e}-\omega_{0}\right) t_{0}-\frac{E_{e}}{E} \sin \varphi$.

Since this differential equation can be integrated, the following relation can be derived to: $\varphi(t)=\frac{\pi}{2}+\arccos \frac{m+f(t)}{1+m f(t)}$,

where $f(t)=\cos \left(\omega_{e}-\omega_{0}\right) \sqrt{1-m^{2}} t$ and $m=E_{e} /\left(E\left(\omega_{e}-\omega_{0}\right) t_{0}\right)$. We find out that $\varphi$ will be constant if $t$ approaches infinity for the case $m^{2}>1$; in this case $\sqrt{1-m^{2}}$ is complex. The value $\varphi(\infty)$ depends on $m$. If $m=1$, we have $\varphi(\infty)=\pi / 2$ and if $m=\infty$ it is $\varphi(\infty)=0$. The cases of a constant phase are corresponding to the entrainment phenomenon. The phase will be increased if $m^{2}$ $<1$ and $m=0$ leads to a linear increasing behavior.

In conclusion we find out by this analysis that in the cases of a constant phase (entrainment) the phase varies between $\varphi= \pm \pi / 2$ and $\varphi=0$ where the concrete value of the asymptotic phase depends on $m$ (that is circuit parameters).

\section{STOCHASTIC ASPECTS OF OSCILLATOR DESIGN}

\section{A. Liouville and Langevin Equation}

In the state space theory of deterministic dynamical systems the traditional approach emphasizes trajectories in the state space $S$ where trajectories can be interpreted as solutions of differential equations with prescribed initial states. In certain cases the trajectory approach of dynamical systems is not useful. Boltzmann observed that the dynamics of sets of initial states instead of single initial state is suitable to calculate interesting physical quantities (see van Kampen [69], chapt. XIV). The deterministic behavior of a system is described by a set of differential equations

$\frac{d x}{d t}=F(x)$,

where $F: \mathfrak{R}^{n} \rightarrow \mathfrak{R}^{n}$. If we are interested in the dynamics of a suitable class of density functions $f: \Re^{n} \rightarrow \mathfrak{R}$ an equation with an associated Frobenius-Perron-Operator $P^{t}$ can be written down

$f(x, t)=P^{t}(f(x))$.

For an explicit representation we interpret the density function $f$ as a probability function which fulfills the following conservation law

$$
\frac{1}{N} \int f(x) d V=1
$$

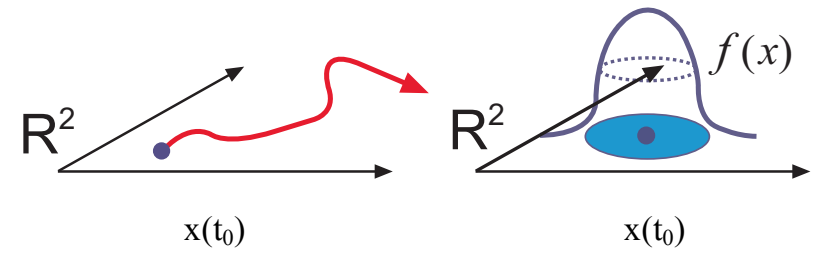

Fig. (10). Types of sets of initial points.

where $d V$ is a suitable measure in the state space of the dynamical system with a suitable normalizing constant $N$. An equivalent formulation of this relation is the continuity 
law and together with the equations of motion (23) the generalized Liouville equation can be derived to:

$$
\frac{\partial f}{\partial t}=-\operatorname{div}\{f \cdot F\}=-\sum_{i=1}^{n} \frac{\partial\left(f \cdot F_{i}\right)}{\partial x_{i}} .
$$

This equation is an equivalent description of deterministic systems if we consider a whole set of initial conditions with a weighting function $f$ instead of a single initial point $x_{0}$ (Fig 10). In the Langevin approach of stochastic systems we start with such a deterministic description and add a stochastic process $\xi$

$\frac{d x}{d t}=F(x)+\sigma(x) \xi$

where the coefficient $\sigma(x)$ characterizes the coupling of the noise source. The first term should be interpreted as a dissipation term where as the second term corresponds to a fluctuation term.

Using the concept of stochastic differential equations $\xi$ has to be interpreted as a generalized white noise process, but in order to solve these equations a more generalized concept of integration (e.g. Kunita [70]) is needed. In essential there are two concepts of stochastic integration which are due to Ito and Stratonovich, respectively, and associated types of stochastic differential equations

$d x=F(x) d t+\sigma(x) d W$,

where $W$ is the so-called Wiener process. Both concepts are mathematicaly equivalent to a Fokker-Planck partial differential equation

$$
\frac{\partial f}{\partial t}=-\sum_{i=1}^{n} \frac{\partial\left(f \cdot F_{i}\right)}{\partial x_{i}}+\sum_{i, j=1}^{n} \frac{\partial^{2}\left(\sigma^{2} f\right)}{\partial x_{i} \partial x_{j}},
$$

which generalizes in some sense the concept of the Liouville equation (26); see also Arnold [71], section 4.2. An alternative approach to the Fokker-Planck equation uses the stochastic Liouville equation and a suitable averaging process; see Kubo [72].

In the case of linear stochastic differential equations - the original subject of Langevin [73] - there is no difference between Ito's and Stratonovich's type. Unfortunately stochastic differential equations (of Ito or Stratonovich type) are sound concepts only from a mathematical point of view if we consider nonlinear Langevin equations. The reason is that each type corresponds to a certain interpretation rule; otherwise its meaning is not well defined. It is interesting to see that for nonlinear Langevin equations in contrast to linear ones the deterministic equation (without noise) does not correspond to the averaged equation (see van Kampen's paper for further details [74])

$\left\langle\frac{d x}{d t}\right\rangle=\frac{d\langle x\rangle}{d t}=\langle F(x)\rangle+\langle\sigma(x) \xi\rangle$.

Even if $\sigma$ is constant and the stochastic part vanishes $(\langle\sigma \xi\rangle=\sigma\langle\xi\rangle=0)$ we note that the function $F$ and the average operator $\langle\cdot\rangle$ do not commute. Only if $\langle F(x)\rangle=F(\langle x\rangle)$ is valid the averaged equation (first moment of the stochastic process $x$ ) fulfills the deterministic equation $\dot{x}=F(x)$. Therefore with van Kampen [75] we come to the conclusion that there is no good reason why the dissipation term should be identical to the vector field of the deterministic equation in nonlinear cases. In order to obtain a sound description of physical systems additional considerations are needed. Based on Stratonovich's ideas Weiss and Mathis [76-78] have presented a substantial work for reciprocal electrical circuits. Since oscillator circuits have to be described by non-reciprocal circuits we use Langevin's approach to construct stochastic equations without a sound physical reasoning for modeling oscillator circuits with noise.

\section{B. Bifurcation Concepts in Noisy Circuits}

In the last section we consider two related concepts for describing general stochastic or noisy circuits and systems if a deterministic description of the form $\dot{x}=F(x)$ is assumed. If we consider non-reciprocal (nonlinear) circuits we have to apply the heuristic Langevin approach where an associated stochastic differential equation can be derived. From a system's theoretical point of view stochastic differential equations belong to the class of state space equations which are formulated in time-domain. An alternative concept of describing noisy circuits uses a probability density function $f$ which satisfies a Fokker-Planck-type equation.

In this section we are concerned with parameterized families of stochastic dynamical systems in the Langevin form $\dot{x}=F(x, \mu)+G(x) \xi$ and its associated Fokker-Planck equation. Although it is known that both concepts are equivalent from a mathematical point of view, it turns out that there are different concepts of stochastic bifurcation. The earlier stochastic bifurcation concept is based on a Fokker-Planck description and was founded in physical applications by Horsthemke and Lefever [79]. In this approach qualitative changing of the stationary solution within the family of Fokker-Planck equations is studied. Although it is a suggestive concept which can be illustrated easily there is no time dependence and therefore it is rather a static concept to bifurcation. In the mathematical literature it is called "P-bifurcation" (e.g. Arnold [80]).

A dynamical concept of stochastic bifurcation is based on the stochastic differential equation. In contrast to the Pbifurcation concept where we look for qualitative changes of the asymptotic probability density function the dynamical (or D-) bifurcation concepts is concerned with qualitative changes of certain properties within the family of stochastic differential equations. For this purpose suitable analogues for equilibrium points of deterministic differential equations is needed. It turns out (see Arnold [80]) that so-called invariant measures of stochastic flows are adequate analogues for deterministic equilibrium points. In doing so we assume that like in the deterministic case a stochastic differential equation is replaced by a "stochastic flow" or so-called cocycle (Arnold [80]). 


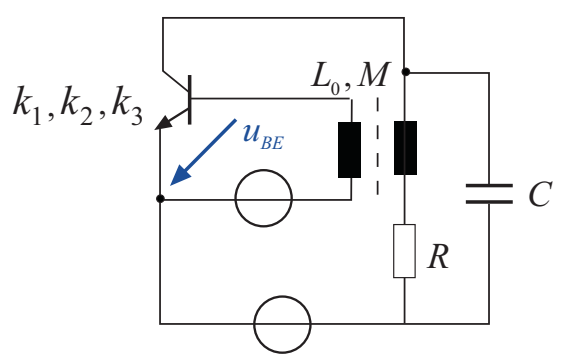

Fig. (11). Transistor meissner oscillator.

Note that if $x_{0}$ is a deterministic equilibrium point of a cocycle $\varphi\left(t, \omega, x_{0}\right)=x_{0}$ then the Dirac measure $\partial x_{0}$ is stationary and invariant. Therefore there is a close relationship of deterministic equilibrium points and invariant measures.

Therefore the fundamental question of D-bifurcation is "Are there other invariant measures than Dirac measures?". It turns out that a necessary condition for qualitative changing in the sense of D-bifurcation is the vanishing of a Lyapunov exponent. It should be mentioned that there is no general relation between P-bifurcation and D-bifurcation.

For illustrating these bifurcation concepts we restrict us to 2-dimensional systems. For higher dimensional systems stochastic concepts for normal forms and/or center manifolds are needed (see Arnold [80]). We consider a Meissner oscillator circuit in Fig. (11). If $k_{2}=0$ the following circuit equation for the voltage between base and emitter can be derived with $\left(\omega_{0}^{2}=1 /(L C)\right)$

$\ddot{u}_{B E}+\left(\frac{R}{L}-\omega_{0}^{2} M\left(k_{1}-3 k_{3} u_{B E}^{2}\right)\right) \dot{u}_{B E}+\omega_{0}^{2} u_{B E}=0$.

Equation (31) can be normalized in the standard van der Pol form $\ddot{x}-\left(\mu-\gamma x^{2}\right) \dot{x}+x=0$. Now we assume with Ariaratnam [81] that we have a noisy resistor which results in an additive decomposition of

$\ddot{x}-\left(\mu_{0}+\sigma \xi-\gamma x^{2}\right) \dot{x}+x=0$.

If (32) is converted into first order equations and polar coordinate transformations are applied to the system, we obtain after a stochastic averaging the following stochastic differential equation for the amplitude process $a(t)$

$d a=\frac{1}{2}\left(\mu_{0}+\frac{5}{8} \sigma^{2}-\frac{1}{4} \gamma a^{2}\right) a d t+\left(\frac{3}{8}\right)^{\frac{1}{2}} a d W_{a}$.

For the analysis of D-bifurcations we have to determine the stability of stationary solutions $a_{s}(t)$ by means of associated Lyapunov exponents. If small variations $r(t)$ of $a_{s}(t)$ are considered the following linearized stochastic differential equation for amplitude process can derived to:

$d r=\frac{1}{2}\left(\mu_{0}+\frac{5}{8} \sigma^{2}-\frac{3}{4} \gamma a_{s}^{2}\right) r d t+\left(\frac{3}{8}\right)^{\frac{1}{2}} \sigma r d W_{a}$.

For the bifurcation analysis the zeros of the Lyapunov exponents have to be found $\lambda=\frac{1}{2}\left(\mu_{0}+\frac{1}{4} \sigma^{2}-\frac{3}{4} \gamma\left\langle a_{s}^{2}\right\rangle\right)$.

Obviously $\lambda$ is zero for the trivial solution $a_{s}=0$ under the condition $\mu_{0}=-\sigma^{2} / 4$. Furthermore for the same value of $\mu_{0}$ we have a zero for $a_{s} \neq 0$, such that we have a Dbifurcation. For studying the P-bifurcation we need a solution of the stationary Fokker-Planck equation associated to the noisy van der Pol equation. It turns out that a first Pbifurcation occurs at $\mu_{0}=\sigma^{2} / 8$ where the peak of the probability density function shifts as $a_{p}:=2\left(\left(\mu_{0^{-}}-\sigma^{2} / 8\right) / \gamma\right)^{1 / 2}$.

Another changing occurs at $\mu_{0}=\sigma^{2} / 2$ where the unimodal density centered at the origin changes to a bi-modal density possessing a ring of peaks; see Ariaratnam [81]. Note that the $\mu_{0}$-values for the $\mathrm{D}$ - and P-bifurcation differ substantially. Additional examples can be found in Arnold [80].

\section{CONCLUSION}

In this paper we considered the essential steps of a systematic concept for the design of electronic oscillator circuits. It can be shown that by means of advanced mathematical methods the intrinsic nonlinear problem of oscillator design is manageable. Using a high-efficient computer algebra system (e. g. MAPLE or MATHEMATICA) semi-analytical expressions for the oscillator design can be derived. Based on our previous work further research will be done in order to develop a computeraided design for electronic oscillators in the $\mathrm{GHz}$ area. It is our goal to enable expert analog designers to design oscillators by means of a variety of well-adapted tools instead of a general purpose circuit simulator.

\section{ACKNOWLEDGEMENTS}

The author would like to thank all his (former) assistants and (former) students who are working in my group since 1985 on oscillator and synchronization aspects. I would like to express my special thanks to Prof. Dr.-Ing. Burkhard Voigt (Hochschule Mannheim), Dr.-Ing. Claudia Keidies (Stadt Dortmund), Dr.-Ing. Laurens Weiss (Qimonda München), Dr.-Ing. Marcus Prochaska (NXP Hamburg), Dipl.-Ing. Kenny Bohle (Cameron GmbH, Celle), Dipl.-Ing. Alexander Belski (Leibniz Universität Hannover), Dipl.-Ing. Judith Schmackers (Bergische Universität Wuppertal), Dipl.Ing. Jens Anders (EPFL Lausanne, Switzerland) as well as can. el. Christopf Zorn, cand. el. Tina Thiessen, cand. el. Jan Przytarski (Leibniz Universität Hannover) for their engaged working in the oscillator analysis and design team. Furthermore my warmest thanks to Prof. Dr. Leon Chua (Unversity of California, Berkeley, USA), Prof. Dr. Ir. Chris Verhoeven (TU Delft), Prof. Dr. John L. Wyatt (MIT, Cambridge, USA), Prof. Dr. Erik Lindberg (Technical University of Denmark, Lyngby), Prof. Dr. Ruedi Stoop (ETH Zürich, Switzerland), Prof. Dr.-Ing. Wolfgang Schwarz (TU Dresden), Prof. Dr. Orla Feely (University College Dublin) and last but not least my friend and coauthor of the John Wiley's EE-RF Handbook article "Oscillator Design” Prof. Dr.-Ing. Peter Russer (TU München). 


\section{REFERENCES}

[1] H. Nicolis and I. Prigogine, Self-Organization in Nonequilibrium Systems: From Dissipative Structures to Order through Fluctuations, New York: John Wiley \& Sons, 1977.

[2] J. Guckenheimer and P. Holmes, Nonlinear Oscillations, Dynamical Systems and Bifurcations of Vector Fields, New York Berlin - Heidelberg - Tokyo: Springer Verlag, 1983.

[3] T. H. O'Dell, Electronic Circuit Design: Art and Practice, New York: Cambridge University Press, 1988.

[4] L. Mandelstam and N. Papalexi, "About resonance phenomena of the frequency division" (in German), Zeitschrift für Physik., vol. 72, pp. 223-248, 1931.

[5] L. Mandelstam, N. Papalexi, A.A. Andronov, S. Chaikin and A. Witt, "Recent results about nonlinear oscillations" (in French), Zeitschr. f. Techn. Physik., vol. 4, pp. 81-134, 1935.

[6] G. M. Maggio, O. De Feo and M. P. Kennedy, "A general method to predict the amplitude of oscillation in nearly sinusoidal oscillators", IEEE Trans. Circ. Syst. I, vol. 51, no. 8, 2004,pp. 1586-1595.

[7] W. Mathis and P. Russer, "Oscillator Design", In: K. Chang, Ed., Encyclopedia of RF and Microwave Engineering, vol. 4, pp. 35633589, New York: John Wiley, 2005.

[8] M. Prochaska, A. Belski and W. Mathis, "Bifurcation Analysis of On-Chip Oscillators", Proc. IEEE Int. Sympos. Circ. Syst. (ISCAS), 2005.

[9] W. Mathis, "Nonlinear Electronic Circuits - An overview", Proc. Conf. Mixed Des. Integr. Circ. Syst. (MIXDES), Poland 2000, pp. 15-17.

[10] W. Duddel, "On rapid variations in the current through the directcurrent arc", Electrician, vol. 46, pp. 269-273 and 330-313, 1900.

[11] H. Th. Simon, "Acoustic phenomena of the electrical arc" (in German), Wiedemanns Annalen der Physik u. Chemie, vol. 64, pp. 233, 1898 .

[12] G. G. Blake, History of Radio Telegraphy and Telephony, London: Radio Press, 1926.

[13] E. Nesper, "On wireless telephoniy" (in German), Elektrotechn. Z., vol. 10, pp. 418-421 and 450-453, 1909.

[14] K.W. Wagner, "The Arc as AC Generator" (in German), Ph.D. Thesis, University of Göttingen, 1910.

[15] J. Zenneck, "About the onset of oscillations using electrical arcs" (in German), Ann. Phys., vol. 43, pp. 481-524, 1914.

[16] W. Mathis, "An Efficient Method for the Transient Analysis of Weakly Damped Crystal Oscillators", Proc. MTNS-98 Symposium, Italy, IL POLIGRAFO, Padova, July 1998, pp. 313-316.

[17] L. Kühn, "On wireless telephony" (in German), Z. Techn. Phys., vol. 3, pp. 109-118, 1922

[18] A. Meißner, "The time of high frequency alternator" (in German), Telefunken-Zeitschrift, vol. 26, pp. 159-164, 1953.

[19] H. R. Johannsen, "The chronology of discoveries and inventions from amber to the microprocessors" (in German), BerlinOffenbach: VDE Verlag, 1986.

[20] L. de Forest, "The Audion", Trans. Am. Inst. Electr. Eng., vol. 25, pp. 735,1906 .

[21] R. V. Lieben, "Cathode ray relay" (in German), DRP 179807 (German Patent), March 4, 1906

[22] R. V. Lieben, E. Reisz and S. Strauß, "Grid between cathode and anode of a Lieben tube, a constant tunable voltage" (in German), DRP 249142 (German Patent), December 20, 1910.

[23] E. H. Armstrong, "Some Recent Developments in the Audion Receiver", Proc. IRE 3, September 1915, pp. 215-247.

[24] A. Meißner, "On tube transmitters" (in German), Elektrotechn. Z, vol. 40, pp. 65-68 and 78-79, 1919.

[25] D.G. Tucker, "The history of positive feedback: the oscillating audion, the regenerative receiver and other applications up to around 1923", Radio Electron. Eng., vol. 42, pp. 69-80, 1972.

[26] G. H. Barkhausen, "The vacuum tube and its technical applications I, II, III" (in German), Jahrbuch der Ddrahtlosen Telegraphie und Telephonie, vol. 14, pp. 27-47, 1919; vol. 16, pp. 82-114, 1920; vol. 18, pp. 402-419, 1921.

[27] K. Skowrennek, "On the development of electron tube amplifiers" (in German), Arch.f. Geschichte Math., d. Naturwiss. u.d. Techn., vol. 13, pp. 225-276, 1931.

[28] F. Paschke, "Walter schottkys first reseach areas" (in German), Siemens Forsch.-u. Entwickl.-Ber., vol. 15, pp. 287-290, 1986.
[29] H. G. Möller, "Quantative treatment of oscillations in tube generators by means of the characteristic of oscillations (Schwingkennlinie)" (in German), Jahrbuch der Drahtlose Telegraphie und Telephonie, vol. 14, pp. 326-360, 1919.

[30] L. A. Hazeltine, "Oscillating audion Circuits", Proc. Inst. Radio Eng., vol. 6, 1918, pp. 63-98.

[31] G. Vallauri, "On the functionality of vacuum tubes with three electrodes (audion) and its application in radiotelegraphy Sul" (in Italian), L'Elettrotecnica, vol. 4, pp. 43-58, 1917.

[32] G. Vallauri, "On the mode of operation of the vacuum tubes with three electrodes (Audion) used in the wireless telegraphy" (in German), Jahrbuch der Drahtlosen Telegraphie und Telephonie 12, pp. 349-364, 1917

[33] R. A. Heising, "The audion oscillator", Phys. Rev., vol. 16, pp. 216-237, 1920

[34] W. Albersheim, "Critical aspects of the problem of pulling and its literature" (in German), Archiv. Elektrotechnik, vol. 14, pp. 23-42, 1924.

[35] J. A. Sanders and F. Verhulst, "Averaging Methods in Nonlinear Dynamical Systems", New York - Berlin - Heidelberg - Tokyo: Springer-Verlag, 1985.

[36] B. van der Pol, "A theory of the amplitude of free and forced triode vibrations", Radio Rev., vol. 1, pp. 701-710, 1920.

[37] G. Joos, "Theory of electron tube oscillators" (in German), Ann. $d$. Phys., vol. 69, pp. 505-547, 1922 .

[38] B. van der Pol, "The nonlinear theory of electric oscillators", Proc. Inst. Radio Eng., vol. 22, 1934, pp. 1051-1089,

[39] M. A. Andronov, "Limit cycles of poincare and the theory of oscillations" (in French), Comptes Rendus (Paris), vol. 189, pp. 559-561, 1929.

[40] A. A. Andronov and A. Witt, "About the mathematical theory of auto-oscillations" (in French), Comptes Rendus (Paris), vol. 190, pp. 256-258, 1930 .

[41] A. A. Andronov and A. Witt, "About the theory of entrainment of van der pol" (in German), Archiv f. Elektrotechnik, vol. 24, pp. 99-110, 1930.

[42] A. A. Andronov, A. Witt and S. Chaikin, Theory of Oscillators (Reprint), New York: Dover Publication Inc., 1966 (first publication 1937)

[43] W. Mathis, Theorie nichtlinearer Netzwerke, Berlin - Heidelberg New York: Springer Verlag, 1987.

[44] C. Bissell, "A.A. Andronov and the Development of Soviet Control Engineering", IEEE Control Syst. Mag., vol. 18, pp. 56-62.

[45] D. Aubin and A.D. Dalmedico, "Writing the History of Dynamical Systems and Chaos: Longue Duree and Revolution, Disciplines and Cultures", Hist. Math., vol. 29, pp. 273-339, 2002.

[46] A. Pechenkin, "The concept of self-oscillations and the rise of synergetics ideas in the theory of nonlinear oscillations", Stud. Hist. Philos. Modern Phys., vol. 33, pp. 269-295, 2002.

[47] N. M. Krylov and N. N. Bogoliubov, "Introduction to Nonlinear Mechanics (in Russian)", Patent No. 1, Kiev 1937.

[48] A. I. Mees and L. O. Chua, "The hopf bifurcation and its application to nonlinear oscillations in circuits and systems", IEEE Trans. Circ. Syst., vol. 26, pp. 235-254, 1979.

[49] M. G. Rosenblum, A. S. Pikovsky, and J. Kurths, "Phase synchronization in driven and coupled chaotic oscillators", IEEE Trans. Circ. Syst., I, vol. 44, no. 10, pp. 874-881, 1997.

[50] J. Pantaleone, "Synchronization of metronomes", Am. J. Phys., vol. 70, pp. 992-1000, 2002

[51] H. G. Möller, The Electron Tube (in German), Braunschweig: Vieweg, 1920

[52] H. G. Möller, "On the undisturbed radio reception with an audion" (in German), Jahrb. f. drahtlose Telegr., vol. 17, pp. 256, 1921

[53] H. Fack, "On the theory of entrainment after van der pol" (in German), Frequenz, vol. 6, pp. 141-145, 1952.

[54] G. Kurz and W Mathis, "Oscillators - Circuits, Analysis and Properties" (in German), Wiesbaden: Hüthig Verlag, 1994.

[55] E. V. Appleton and B. van der Pol, "On a type of oscillationhysteresis in a single triode generator", Phil. Mag., vol. 43, pp. 177, 1922.

[56] W. Mathis, "Historical remarks to the history of electrical oscillators", Proc. MTNS-98 Symposium, Padova 1998, pp. 309312.

[57] P. Rjasin, "Pulling and beating processes of entrainment" (in German), J. Tech. Phys. URSS, vol. 2, pp. 195-214, 1935. 
[58] J. W. S. Rayleigh, Collected Papers, Cambridge, vol. V, p. 369, vol. I, p. 409, 1899-1920.

[59] S. Chaikin, "Quantitative untersuchung der akustischen mitnahmeerscheinung", Elektr. Nachrichtentech.(ENT), vol. 9, pp. 376-382, 1932.

[60] H. de Bellescise, "Die reception of synchronization" (in French), On de Electrique, vol. 11, pp. 230-240, 1932.

[61] F. Kirschstein, "The entrainment of oscillations and its technical applications" (in German), Elektr. Nachrichtentech. (ENT), vol. 20, pp. 29-38, 1943.

[62] R. Urtel, "Entrainment and synchronization of oscillations" (in German), Z. Techn. Phys., vol. 11, pp. 460-465, 1938.

[63] W. Mathis, "Transformation and Equivalence", in The Circuits and Filters Handbook, W.-K. Chen, Ed., Boca Raton: CRC Press \& IEEE Press, 2003.

[64] J. Bremer, C. Zorn and W. Mathis, "Bifurcation Analysis of an LCTank VCO Including the Variable Capacitance", Proc. Conference for Mixed Design of Integrated Circuits and Systems (MIXDES), pp. 389- 394, Poznan 2008.

[65] R. Bunch and S. Raman, "Large-signal analysis of MOS varactors in CMOS -Gm LC VCOs", IEEE J. Solid-State Circ., vol. 38, no.8, 2003.

[66] M. Hershenson, A. Hajimiri, S. Mohan, S. Boyd and T. Lee, "Design and optimization of LC oscillators", Proceedings IEEE/ACM international conference on Computer-aided design, pp. 65-69, 1999.

[67] U. Kirchgraber and E. Stiefel, "Methods of the Analytical Perturbation and its Applications" (in German), Teubner-Verlag 1978.

[68] E. J. Cassignol, Semiconductors, Vol. III (Non-linear Electronics)", Eindhoven, The Netherlands: Philips Technical Library, 1968.
[69] N. G. van Kampen, Stochastic Processes in Physics and Chemistry, Amsterdam: North Holland, 1992.

[70] H. Kunita, "Stochastic flows and stochastic differential equations", New York: Cambridge University Press, 1990.

[71] L. Arnold and P. Imkeller, "Normal forms for stochastic differential equations", Probab. Theory Relat. Fields, vol. 110, pp. 559-588, 1998.

[72] R. Kubo, "Stochastic liouville equations", J. Math. Phys., vol. 4, pp. 174-183, 1963 .

[73] P. Langevin, "On the Theory of brownian movement" (in French), Comptes Rendus Acad. Sei. (Paris), vol. 146, pp. 530, 1908.

[74] N. G. van Kampen, "The validity of nonlinear langevin equations", J. Stat. Phys., vol. 25, pp. 431-442, 1981.

[75] N. G. van Kampen, "Thermal fluctuations in nonlinear systems", J. Math. Phys., vol. 4, pp. 190-194, 1963.

[76] L. Weiss and W. Mathis, "Irreversible thermodynamics and thermal noise of nonlinear networks", Int. J. Comput. Math. Electr. Electron. Eng., vol. 17, pp. 635-648, 1998.

[77] L. Weiss and W. Mathis, "A thermodynamical approach to noise in nonlinear networks", Int. J. Circ. Theor. Appl., vol. 26, pp. 147165,1998

[78] L. Weiss, "Noise in nonlinear electronic circuits and devices - a thermodynamic Approach" (in German), Ph.D. Thesis, Univ. Magdeburg, Berlin-Offenbach: VDE Verlag, 1999.

[79] W. Horsthemke and R. Lefever, Noise-Induced Transitions, Berlin-Heidelberg-New-York: Springer Verlag, 1984.

[80] L. Arnold, Random dynamical systems, Berlin - Heidelberg New York: Springer Verlag, 1998.

[81] S. T. Ariaratnam, "Stochastic Bifurcation in Hereditary Systems", Proc. 8th ASCE Spec. Conference on Prob. Mech. Struct. Reliability, Notre Dame University, USA, July 2000.

\section{About the Authors}

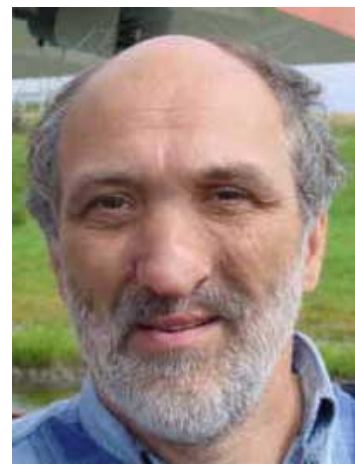

Wolfgang Mathis was born in Celle (Germany) on May 13, 1950. He received the Dipl.-Phys. degree in physic in 1980 and the Dr.- Ing. degree, (Ph.D.) in electrical engineering in 1984, both from the Technical University of Braunschweig. He was granted the Habilitation degree from the University of Braunschweig in 1988 for Network Theory and Computer Aided-Design. He became a full professor at the University of Wuppertal (1990) and Magdeburg (1996). Since 2000 he is a full professor at the University of Hannover and holds the chair of Electromagnetic Theory (TET). He was a visiting researcher at the University of California, Berkeley (USA) and Lodz (Poland). His research interests include theory of nonlinear circuits and dynamical systems, theory of nonlinear noisy networks and systems (incl. its thermodynamic theory), computer-aided circuit analysis and design, realization of nonlinear circuits (oscillators, PLL, class-D power amplifiers), numerical mathematics, parallel computation, nanoelectronics, quantum computing, analysis and numerics of electromagnetic fields, computer algebra, and history of Electrical Engineering and Technical Physics. Furthermore he is chair of the IEEE Circuits and Systems Society German chapter since 2001. He has become an IEEE senior member since 1998 and he received the IEEE Fellow award in 1999. He is a member of IEEE, VDE/ITG, DPG (German Physical Society) as well as of the Academy of Science and Art Nordrhein-Westfalen and the German Academy of Science and Engineering (acatech). 


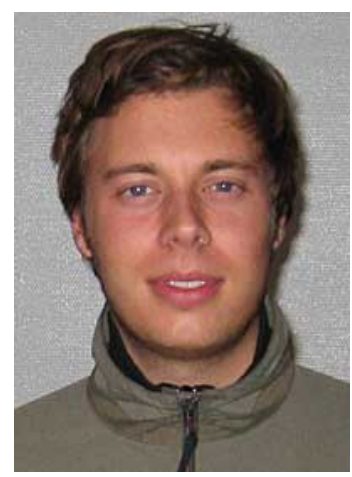

Jan Bremer received his Dipl.-Ing degree in electrical engineering from the Leibniz University of Hanover (Germany) in 2006. Since that time he is working as a research engineer at the Institute of Electromagnetic Theory at the Leibniz University of Hanover where he is working towards the Ph.D. degree. His main research interests are the systematic design of analog RF circuits with a focus on oscillator circuits and computer-aided circuit design. Jan Bremer can be contacted at: bremer@tet.uni-hannover.de 